\title{
A Large Retinal Capillary Hemangioma in the Anterior Retina Treated with Photodynamic Therapy
}

\author{
Janice Cheung Gary K.Y. Lee Clement C.Y. Tham
}

Department of Ophthalmology and Visual Sciences, The Chinese University of Hong Kong, Hong Kong, SAR, China

\section{Key Words}

Retinal capillary hemangioma · Photodynamic therapy · Subretinal fluid

Abstract

Purpose: To report a case of a large anterior retinal capillary hemangioma $(\mathrm{RCH})$ treated successfully with photodynamic therapy (PDT).

Methods: Case report.

Results: A 12-mm-large $\mathrm{RCH}$ located in the anterior retina, with vision-threatening exudative complications that had not responded to cryotherapy and repeated laser photocoagulations, was treated with PDT using verteporfin. Exudation regressed and tumor growth arrested after treatment.

Conclusion: PDT can be delivered effectively to a lesion in the anterior retina and should be included as an option for treating anteriorly located $\mathrm{RCH}$ when conventional cryotherapy and laser photocoagulation fail.

\section{Introduction}

Retinal capillary hemangiomas (RCH) are uncommon benign vascular tumors of the retina. Small peripheral RCH can usually be treated successfully by laser photocoagulation of the lesion and the afferent arteriole, with or without cryotherapy. Effective treatment using photodynamic therapy (PDT) for lesions in the posterior pole has also been documented [1]. We report a case of a large peripheral RCH treated with PDT after treatment with laser and cryotherapy had failed. To our knowledge, this is the first reported use of PDT in an anteriorly located RCH not associated with von Hippel-Lindau disease. 


\section{Case Report}

A 29-year-old female presented with a 1-month history of blurred vision in her right eye. At presentation, her best-corrected visual acuity (BCVA) was 20/40. Examination showed a grade-1 epiretinal membrane at the macula and a small RCH in the peripheral retina near the ora serrata at 12 o'clock (fig. 1a). Systemic investigations showed no evidence of von Hippel-Lindau disease. BCVA improved to 20/28 after surgical removal of the epiretinal membrane. One year after the operation, her BCVA dropped to 20/100. There was a significant increase in the size of the RCH (largest diameter up to $12 \mathrm{~mm}$ ), with the surrounding subretinal fluid (SRF) and exudation extended to the macula (fig. $1 \mathrm{~b}, \mathrm{c})$. A posterior subcapsular cataract was also noted. Subsequently, the patient underwent phacoemulsification with intra-ocular lens implantation and cryotherapy to the RCH. Repeated sessions of focal laser ablation to the feeder vessels were performed but they failed to control the exudation. After discussion of the treatment options with the patient, standard PDT was performed using a verteporfin infusion at a dose of $6 \mathrm{mg} / \mathrm{m}^{2}$ body surface area for $10 \mathrm{~min}$. Five minutes after completion of the infusion, light exposure was performed with a diode laser $(689 \mathrm{~nm})$ at an intensity of $600 \mathrm{~mW} / \mathrm{cm}^{2}$. Using a Volk SuperQuad ${ }^{\circledR} 160$ diode laser lens, the whole RCH was covered with two successive confluent burns with spot sizes measuring 6,400 and 6,200 $\mu \mathrm{m}$, respectively. Laser was applied for $83 \mathrm{~s}$ for each treatment spot (power: $50 \mathrm{~J} / \mathrm{cm}^{2}$ ). Two days after PDT, there was a significant increase in the surrounding SRF that resulted in exudative detachment of the superior retina and macula. The exudation resolved spontaneously in 5 days. Five months after PDT, the SRF surrounding the RCH resolved completely with a reduction in size of the RCH (fig. 2 ). The final BCVA in this patient was $20 / 20$.

\section{Discussion}

Previously, PDT had been reserved for treating RCH in the posterior retina and peripapillary region when laser photocoagulation and cryotherapy were considered ineffective or unsafe to apply [2]. Due to the original design of PDT for the treatment of macular choroidal neovascularization, the dynamic field of view of commercially available PDT laser lenses is within the range of $133-137^{\circ}$, which is not adequate to cover lesions in the extreme periphery. As a result, laser photocoagulation and cryotherapy have remained treatment options for peripherally located RCH in most cases. When such conventional treatments are ineffective, more aggressive modalities, such as radioactive plaque therapy or surgical excision, would need to be considered [3, 4]. Our case has demonstrated that PDT can be delivered effectively to a lesion in the anterior retina using a wide-field diode laser contact lens. The positive treatment response was evidenced by tumor shrinkage and resolution of exudation. Transient increase in exudation from RCH following PDT has been documented previously [5]. Such exudation is usually self-limiting in nature and had no effect on the final visual outcome in our case. In summary, our case has illustrated that PDT is an effective treatment for large RCH in the anterior retina when conventional laser and cryotherapy have failed to control the disease.

\section{Disclosure Statement}

The authors have nothing to disclose. 

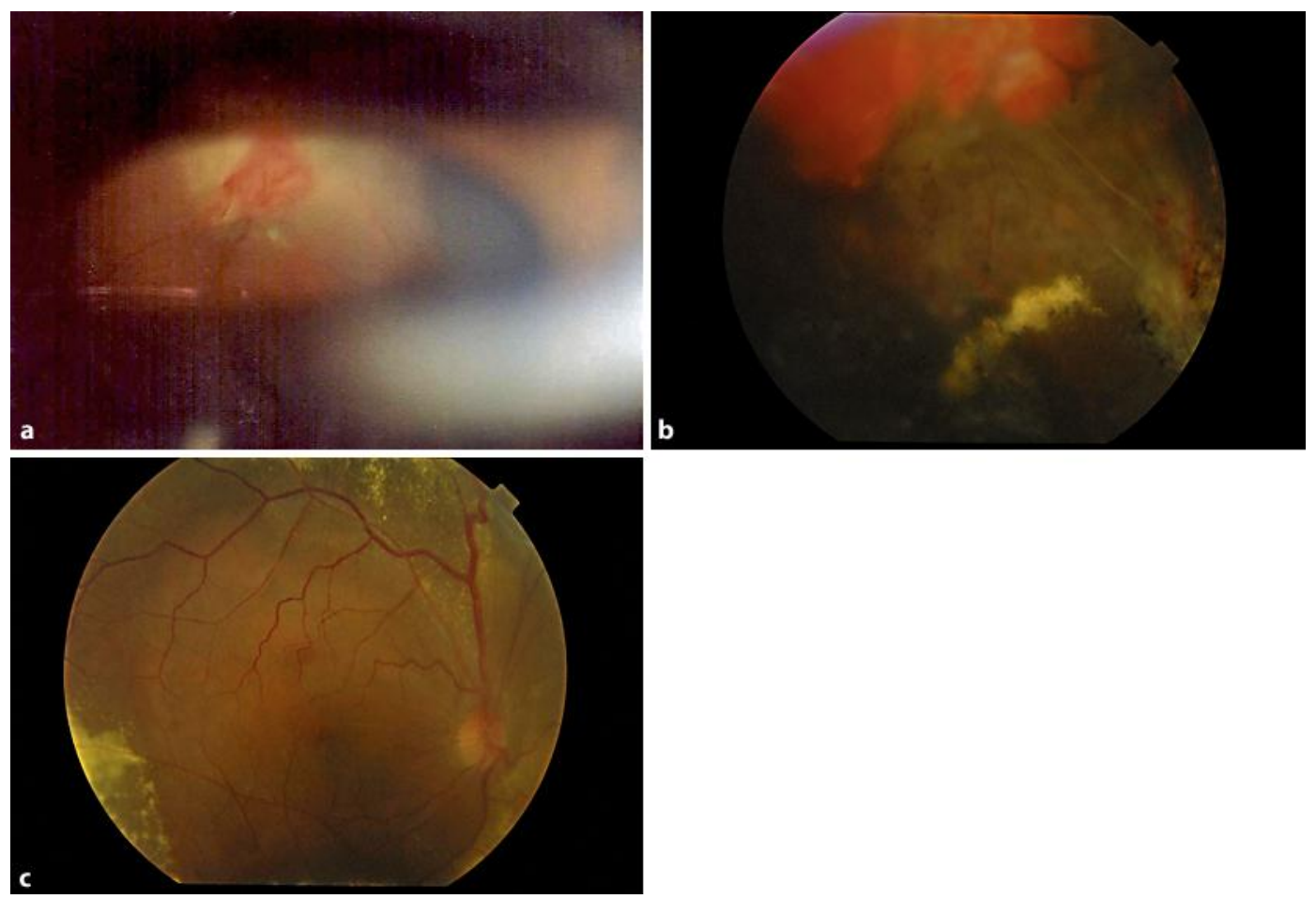

Fig. 1. a Appearance of the RCH at presentation when examined under a Goldmann 3-mirror lens. It was located near the ora serrata with minimal surrounding exudates. $\mathbf{b}$ Fundus photo of the right eye shows the subsequent enlargement of the RCH and the associated increase in the amount of SRF and exudates. The largest diameter of the RCH measured up to $12 \mathrm{~mm}$. c SRF and exudates from the RCH extended to involve the macula.

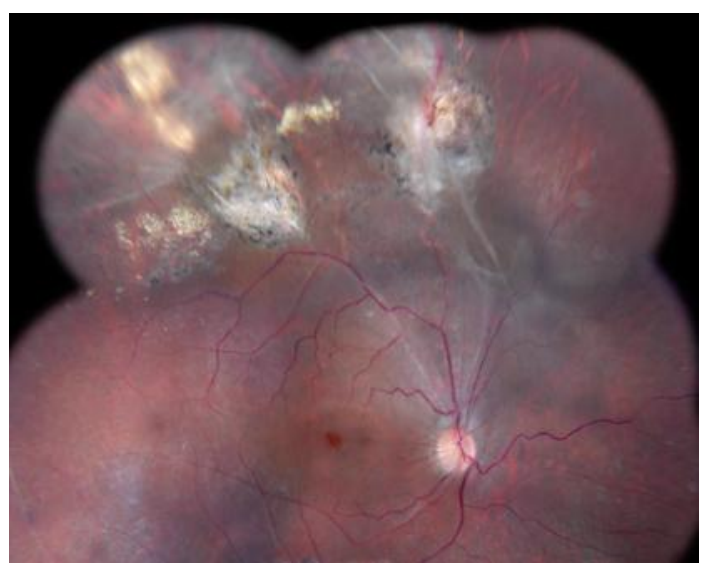

Fig. 2. Fundus photo taken 5 months after PDT shows the complete resolution of SRF and exudates. Chorioretinal scars from previous laser photocoagulation of the feeder vessels can be seen in the superior quadrant. The RCH reduced in size and cannot be visualized in the area covered in this photo. 


\section{References}

1 Sachdeva R, Dadgostar H, Kaiser PK, Sears JE, Singh AD: Verteporfin photodynamic therapy of six eyes with retinal capillary haemangioma. Acta Ophthalmol 2010;88:e334-e340.

-2 Singh AD, Nouri M, Shields CL, Shields JA, Perez N: Treatment of retinal capillary hemangioma. Ophthalmology 2002;109:1799-1806.

3 Kreusel KM, Bornfeld N, Lommatzsch A, Wessing A, Foerster MH: Ruthenium-106 brachytherapy for peripheral retinal capillary hemangioma. Ophthalmology 1998;105:1386-1392.

4 Gaudric A, Krivosic V, Duguid G, Massin P, Giraud S, Richard S: Vitreoretinal surgery for severe retinal capillary hemangiomas in von Hippel-Lindau disease. Ophthalmology 2011;18:142-149.

5 Schmidt-Erfurth UM, Kusserow C, Barbazetto IA, Laqua H: Benefits and complications of photodynamic therapy of papillary capillary hemangiomas. Ophthalmology 2002;109:1256-1266. 